\title{
Conditional Expression of a Myocardium- Specific Transgene in Zebrafish Transgenic Lines
}

\author{
Chiu-Ju Huang, ${ }^{\dagger \dagger}$ Tzuu-Shuh Jou, ${ }^{2 \dagger}$ Yi-Lwun Ho, ${ }^{2}$ Wu-Hsun Lee, ${ }^{1}$ Yu-Ting Jeng, ${ }^{1}$ Fong-Jou Hsieh,,${ }^{2,3}$ \\ and Huai-Jen Tsai ${ }^{1,3 *}$
}

To develop the first heart-specific tetracycline (Tet)-On system in zebrafish, we constructed plasmids in which the cardiac myosin light chain 2 promoter of zebrafish was used to drive the reverse Tet-controlled transactivator (rtTA) and the green fluorescent protein (GFP) reporter gene was preceded by an rtTAresponsive element. In the zebrafish fibroblast cell-line, rtTA-M2, one of rtTA's derivatives, demonstrated the highest increase in luciferase activity upon doxycycline (Dox) induction. We then generated two germ lines of transgenic zebrafish: line T03 was derived from microinjection of a plasmid containing rtTA-M2 and a plasmid containing a responsive reporter gene, whereas line T21 was derived from microinjection of a single dual plasmid. Results showed that line T21 was superior to line T03 in terms of greater GFP intensity after induction and with of minimal leakiness before induction. The photographic images of induced GFP in the heart of F2 larvae showed that the fluorescent level of GFP was dose-responsive. The level of GFP expressed in the F3 3 days postfertilization larvae that were treated with Dox for $1 \mathrm{hr}$ decreased gradually after the withdrawal of the inducer; and the fluorescent signal disappeared after 5 days. The GFP induction and reduction were also tightly controlled by Dox in the F3 adult fish from line T21. This Tet-On system developed in zebrafish shows much promise for the study of the gene function in a specific tissue at the later developmental stage. Developmental Dynamics 233:1294-1303, 2005. $\odot 2005$ Wiley-Liss, Inc.

Key words: zebrafish; heart-specific; inducible; Tet-On; cardiac myosin light chain 2

Received 14 October 2004; Revised 15 February 2005; Accepted 4 March 2005

\section{INTRODUCTION}

Zebrafish (Danio rerio) is an excellent animal model in the study of cardiac research (Poss et al., 2002; Raya et al., 2003; Shu et al., 2003). Zebrafish has a two-chambered heart that is similar in many ways to the three- or fourchambered hearts of higher vertebrates (Fishman and Stainier, 1994; Fishman and Chien, 1997). Transpar- ent embryos make it possible to observe the dynamic expression of the heart gene in a noninvasive manner. Embryos that receive oxygen through diffusion will result in embryos with cardiac defects, and these embryos can survive for 1 week (Sehnert et al., 2002). Furthermore, many heart mutant forms are available. However, some heart diseases occur at later de- velopmental stages or even in adulthood. The conventional approaches, i.e., by injection of mRNA for the study of overexpression or by the injection of morpholino for knockdown study, are carried out in one-celled fertilized eggs. This system makes it difficult to generate a heart disease model in some cases. Therefore, we developed a tetracycline (Tet) -depen-

\footnotetext{
${ }^{1}$ Institute of Molecular and Cellular Biology, National Taiwan University, Taipei, Taiwan

${ }^{2}$ Departments of Internal Medicine, and Obstetrics and Gynecology, National Taiwan University Hospital, Taipei, Taiwan

${ }^{3}$ Angiogenesis Research Center, National Taiwan University, Taipei, Taiwan

Grant sponsor: National Science Council of Taiwan; Grant number: NSC93-2313-B002-058: Grant number: NSC93-3112-B-002-32; Grant sponsor: the National Taiwan University Hospital; Grant number: 92A-1002.

Drs. Huang and Jou contributed equally to this work.

*Correspondence to: Huai-Jen Tsai, Institute of Molecular Cellular Biology, No. 1, Section 4, Roosevelt Road, Fisheries Science Building, National Taiwan University, Taipei 106, Taiwan, ROC. E-mail: hjtsai@ntu.edu.tw
}

DOI 10.1002/dvdy.20485

Published online 23 June 2005 in Wiley InterScience (www.interscience.wiley.com). 


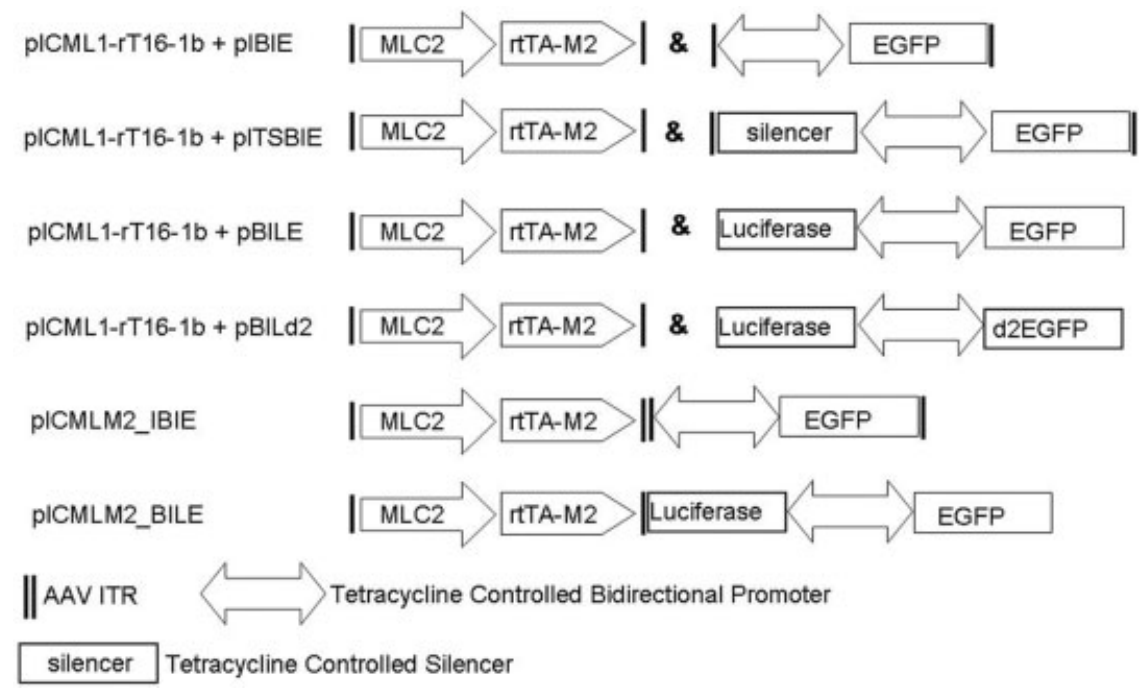

Fig. 1. Schematic illustration of the plasmid constructs used in this study. One-cell stage fertilized zebrafish eggs were microinjected with the cardiac-specific rtTA plasmid pICML1-rT16-1b, in which rtTA-M2 is driven by a zebrafish cm/c2 promoter (MLC2) and flanked by AAV-ITR (black vertical bars), and a tetracycline (Tet) -responsive plasmid, in which reporter gene(s) is driven by a Tet-controlled bidirectional promoter with or without flanking AAV-ITR. Silencer, Tet-controlled transcriptional silencer; d2EGFP; unstable enhanced green fluorescent protein (EGFP). The pICMLM2_IBIE and pICMLM2_BILE are single dual plasmids derived from cointegration of pICML1-rT16-1b and pIBIE, or pICML1-rT16-1b and pBILE, respectively.

dent transcriptional activation system in zebrafish, in which the transgene can be induced to express specifically in the heart at any stage of development.

By taking advantage of the regulatory loop of tet resistance in prokaryotes, tight control of gene expression can be accomplished by titrating the dose of Tet (Gossen and Bujard, 1992) or its more stable derivative doxycycline (Dox; Gossen et al., 1995). By fusing the Tet repressor (TetR) and activating the transcription domain of the virion protein 16 of the herpes simplex virus, Tet-controlled transactivator (tTA) is generated (Gossen and Bujard, 1992). In the absence of Tet, tTA can stimulate $\mathrm{P}_{\text {tet }}-1$, a minimal promoter fused to the tet operator (tet $\mathrm{O})$, and, thus, can activate downstream genes. This approach is referred to as the Tet-Off system, because the binding affinity between TetR and tet $\mathrm{O}$ is allosterically affected by Tet. A Tet-On system is developed by mutagenesis, substituting four amino acids in tTA to generate reverse tTA (rtTA; Gossen et al., 1995), which requires Tet for binding to tet $\mathrm{O}$ and the subsequent activation of the downstream gene. For pharmacokinetic reasons, controlling genes by means of rtTA is of particular advan- tage in the study of transgenic animals. Whenever a gene has to be kept silent, the Tet-On system is regarded as more useful than the Tet-Off alternative in developmental biology studies and gene therapy (Jost et al., 1997).

Both tTA and rtTA systems have been used in studies of gene function in various cellular systems (Salucci et al., 2002; Zhu et al., 2002), as well as in whole organisms (Meissner et al., 2001), such as Toxoplasma gondii (Nakayama et al., 2000), fungus (Weinmann et al., 1994), plants (Furth et al., 1994), and mice (Passman and Fishman, 1994; Kistner et al., 1996; Yu et al., 1996; Jost et al., 1997). However, these inducible expression systems have not been established in zebrafish.

In the previous study, we demonstrated that a $1.6-\mathrm{kb}$ regulatory region of zebrafish cardiac myosin light chain 2 gene $(\mathrm{cmlc} 2)$ in plasmid pICMLE-(-870/787) specifically drives green fluorescent protein (GFP) expression in the heart of transgenic zebrafish (Huang et al., 2003). In this report, we used this segment of $c m l c 2$ to direct rtTA in the Tet-On system and also generated germ-line transgenic zebrafish carrying this heart-specific Tet-On system. The dose-response dependency was analyzed, and the kinetics of Dox-mediated activation of GFP expression was measured. This article is the first report of a zebrafish Tet-On system. We propose that it will be useful for the study of gene function in heart development and for animal modeling of heart disorders.

\section{RESULTS}

\section{Efficiency of Various Transcativators in the Cell Line}

To evaluate the function of various transactivators in a zebrafish system, the fibroblast cell line was transfected with the DNA constructs expressing various transactivators together with the Tet-regulated luciferase plasmid (Fig. 1). Because the codon usage bias had been proposed as being an obstacle in establishing a Tet-inducible system in Toxoplasma (Nakayama et al., 2000), we also compared the transactivation efficiency between tTA, rtTA, rtTA-M2, and rtTA-S2 of prokaryotic origin and the humanized codons rtTA2 ${ }^{\mathrm{S}}-\mathrm{S} 2$ and $\mathrm{rtTA} 2^{\mathrm{S}}-\mathrm{M} 2$. The results are shown in Table 1 . All transactivators displayed a regulated capacity of activating luciferase expression. Among them, rtTA-M2 and the humanized rtTA2 ${ }^{\mathrm{S}}$-S2 mutant had the best performance in terms of increase of luciferase activity after induction. Although rtTA2 ${ }^{\mathrm{S}}$-S2 displayed the least leakiness before induction, the reporter activity was one order of magnitude less than that of the rtTA-M2

\section{Transient Expression of Induced GFP in Embryos Microinjected With Two Separate Plasmids}

To determine the conditional expression of GFP in vivo, we microinjected the zebrafish embryos with the upstream plasmid pICML1-rT16-1b, in which rtTA-M2 was driven by a heartspecific promoter, and the downstream reporter plasmid pIBIE, pBILE, pBILd2, or pITSBIE. Both the background and the induced heartspecific GFP expression were monitored. As shown in Table 2, when 
TABLE 1. Doxycycline-Dependent Activation of Luciferase by tTA, rtTA and Its Derivatives in Zebrafish Fibroblast Cells ${ }^{\text {a }}$

\begin{tabular}{|c|c|c|c|}
\hline \multirow[b]{2}{*}{ Transactivators } & \multicolumn{2}{|c|}{ Luciferase activity } & \multirow[b]{2}{*}{ Activation folds } \\
\hline & - Dox & + Dox & \\
\hline tTA & $10916.4 \pm 1001.2$ & $1571.9 \pm 170.3$ & 6.94 \\
\hline rtTA & $2630.5 \pm 211.8$ & $7979.9 \pm 955$ & 3.03 \\
\hline rtTA-S2 & $2454.8 \pm 236.3$ & $17368.1 \pm 757.7$ & 7.08 \\
\hline rtTA-M2 & $2617.4 \pm 206.3$ & $22440.7 \pm 393.9$ & 8.57 \\
\hline rtTA2 $^{\mathrm{s}}-\mathrm{S} 2$ & $219.2 \pm 33.8$ & $2010.0 \pm 101.3$ & 9.17 \\
\hline rtTA2 $2^{\mathrm{s}}-\mathrm{M} 2$ & $904.1 \pm 126.2$ & $2787.4 \pm 285.7$ & 3.08 \\
\hline \multicolumn{4}{|c|}{$\begin{array}{l}\text { aZebrafish fibroblast cell line ZEM2S was transfected with plasmids expressing their respective transactivators. The upstream } \\
\text { plasmids were controlled by a CMV promoter, and the downstream plasmids contained a luciferase reporer driven by various } \\
\text { Tet-responsive elements. After transfection, cells were divded in two groups and were grown in the absence or presence of } 1 \mu \mathrm{g} / \mathrm{ml} \\
\text { of Dox for } 40 \mathrm{hr} \text { before dual luciferase assays were performed. The values of luciferase activity were arbitrary light units, and fold } \\
\text { activation was determined by dividing the values of the induction by the noninduced conditions. } \\
\text { Dox, doxycycline. }\end{array}$} \\
\hline
\end{tabular}

TABLE 2. Leakiness, Intensity and Heart-Specificity of GFP in the Transgenic Embryos Before and After Induction*

\begin{tabular}{|c|c|c|c|c|}
\hline & \multicolumn{4}{|c|}{ GFP expression } \\
\hline & \multicolumn{2}{|c|}{-Dox (non-specific expression) } & \multicolumn{2}{|c|}{ +Dox (heart-specific expression) } \\
\hline & $\%(\text { Number })^{\mathrm{a}}$ & Intensity $^{\mathrm{b}}$ & $\%(\text { Number })^{\mathrm{c}}$ & Intensity $^{\mathrm{b}}$ \\
\hline pICML1-rT16-1b+pIBIE & $38(90 / 235)$ & $* * *$ & $13(24 / 186)$ & $* * * * *$ \\
\hline pICML1-rT16-1b+pBILE & $12(35 / 287)$ & $* *$ & $11(26 / 247)$ & $* * * *$ \\
\hline pICML1-rT16-1b+pBILd2 & $0(0 / 244)$ & - & $3(6 / 173)$ & $*$ \\
\hline pICML1-rT16-1b+pITSBIE & $24(19 / 79)$ & $* *$ & $10(3 / 33)$ & $* * *$ \\
\hline pICMLM2_IBIE ${ }^{\#}$ & $35(67 / 184)$ & $* * *$ & $23(38 / 165)$ & $* * *$ \\
\hline pICMLM2_BILE ${ }^{\#}$ & $20(112 / 554)$ & $*$ & $16(67 / 415)$ & $* * * *$ \\
\hline \multicolumn{5}{|c|}{$\begin{array}{l}\text { *Before induction, the transferred 72-hours postfertilization (hpf) embryos were examined for leakiness of green fluorescence. The } \\
\text { nonleaky } 72 \text {-hpf embryos were collected and green fluorescence protein (GFP) expression was induced by adding Dox at a } \\
\text { concentration of } 10 \mu \mathrm{g} / \mathrm{ml} \text { for } 72 \mathrm{hr} \text {. The level and specific expression of GFP were then observed at } 144 \text { hpf. } \\
\text { aThe number of surviving transgenic embryos expressing GFP either in the heart or in any part of body before induction. } \\
\text { bThe level of GFP intensity was based on optical observations: *****, the highest expression; } \text {, the lowest expression; -, not } \\
\text { detectable. } \\
\text { cThe number of nonleaky transgenic embryos having heart-specific expression of GFP after induction. } \\
\text { GFP, green fluorescent protein. }\end{array}$} \\
\hline
\end{tabular}

pICML1-rT16-1b was co-microinjected with pIBIE, containing EGFP and flanked by AAV-ITR, the embryos showed that the leak rate $(38 \%)$ and leakiness level (***) reached their highest values. When nonleaky embryos were selected and treated with Dox, we found that the intensity of the heart-specific GFP expression increased dramatically from no expression up to the highest expression level (*****). The heart-specific expression rate after induction for the $186 \mathrm{em}$ bryos examined was $13 \%$ of 186 nonleaky transgenic embryos.

In the experiment of using pBILE, in which AAV-ITR was absent, the leak rate was as low as $12 \%$ of 287 embryos, which was only one third of the rate for the embryos injected with pIBIE. The Dox-induced heartspecific expression rate of pBILE-injected embryos was $11 \%$, which was quite close to that of the pIBIE-injected embryos. In addition, the level of GFP intensity also increased greatly from no expression up to $* * * *$ after induction.

When pBILd2, in which enhanced GFP (EGFP) in pBILE was replaced by d2EGFP, was used as the downstream plasmid, there was no leaky expression in the transgenic embryos. However, the rate of heart- specific induced GFP was as low as $3 \%$ for 173 injected embryos, and the intensity of the induced fluorescent signal was relatively faint after induction.

When pICML1-rT16-1b was co-microinjected with plasmid pITSBIE, in which a transcriptional silencer was included, it was found that the leaky expression was not effectively suppressed. In addition, we noticed that only as few as $10 \%$ of embryos exhibited heart-specific induced fluorescence and a moderate increase in GFP intensity after induction. In conclusion, co-microinjection of plasmids pICML1-rT16-1b and pIBIE or 
A
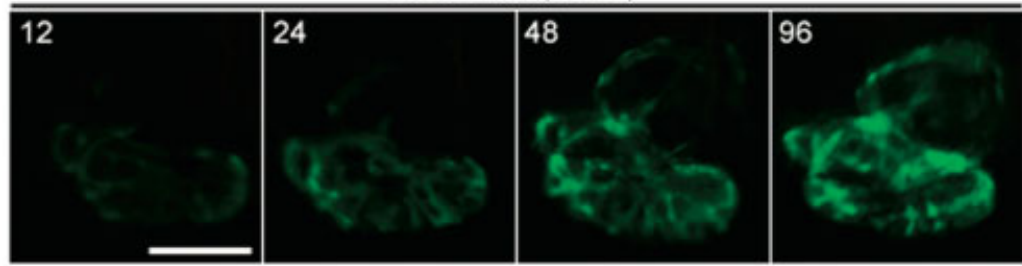

B
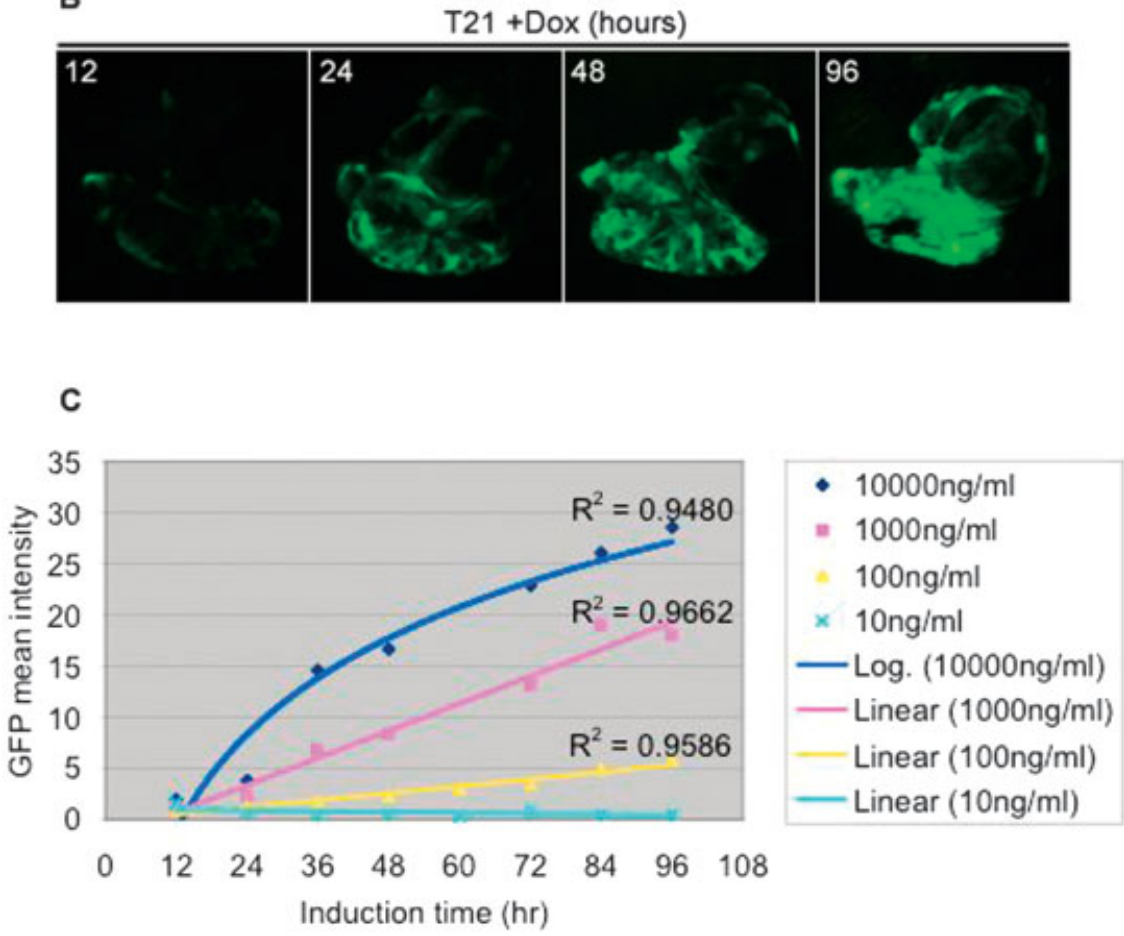

Fig. 2. The doxycycline (Dox) -induced green fluorescent protein (GFP) expression in the zebrafish F2 larvae derived from transgenic lines. A,B: The photographic images of GFP intensity were taken at 12-hr intervals after the 3-days postfertilization (dpf) larvae from lines T03 (A) and T21 (B) were exposed to $10 \mu \mathrm{g} / \mathrm{ml}$ Dox. C: Dose-response and kinetics of GFP induction occurred in line T03 were analyzed. The mean values of GFP intensity after induction with 10,000,1,000,100, and 10 $\mathrm{ng} / \mathrm{ml}$ Dox were analyzed at 12-hr intervals until the end of $96 \mathrm{hr}$. A detailed analysis of the GFP intensity is described in the Experimental Procedures section. $\mathrm{R}^{2}$ represents either logarithm or linear regression coefficient. Scale bars $=0.1 \mathrm{~mm}$ in $A, B$
A

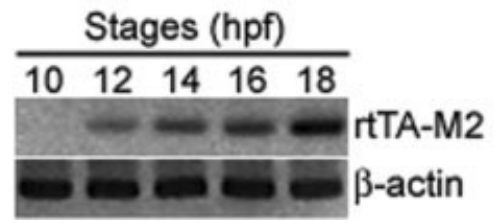

B

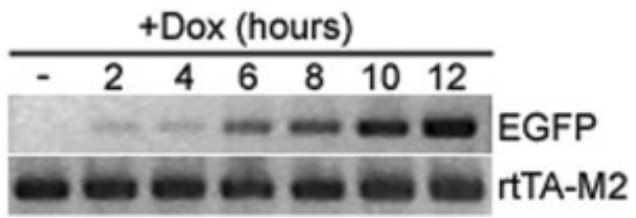

Fig. 3. Detection of the transcripts of rtTA and green fluorescent protein (GFP) genes in the F3 embryos by using semiquantitative reverse transcription-polymerase chain reaction. A: Detection the existence of rtTA-M2 transcripts every $2 \mathrm{hr}$ starting from $10 \mathrm{hr}$ postfertilization (hpf) embryos before induction. The rtTA-M2 transcripts were detectable from 12-hpf, and the signals reached a normal level at 18 -hpf. Detection of $\beta$-actin transcripts served as an internal control. B: Detection of the existence of GFP transcripts before and after the 3-dpf embryos were treated with doxycycline (Dox). The rtTA-M2 transcripts were detectable, whereas the GFP transcripts were detectable $2 \mathrm{hr}$ later after Dox induction.
pBILE was the most effective construct in this study in terms of lower leakiness before induction and higher fluorescent intensity after induction.

\section{Transient Expression of Induced GFP in Embryos Microinjected With a Single Dual Plasmid}

The highest competence of transactivation was obtained from embryos microinjected with pICML1-rT16-1b and a combination of pIBIE or pBILE (Table 2). Thus, we constructed the plasmids pICMLM2_IBIE and pICMLM2_BILE, which were the cointegrated forms of the upstream plasmid pICML1-rT16-1b and the downstream plasmid pIBIE or pBILE, respectively.

When comparing the transactivation potency of embryos microinjected with the separated forms pICML1-rT16-1b and pIBIE with that of the embryos injected with the cointegrated form pICMLM2_IBIE, it was found that the leak rate and intensity were similar (Table 2). After Dox induction, the intensity of the heart-specific GFP expression in the embryos injected with the cointegrated form of plasmid was much less than that in those injected with the separated forms, despite that the induction rate was as high as $23 \%$ of 165 embryos (Table 2). Meanwhile, the leak rate of pICMLM2_BILEinjected embryos was higher than that of the embryos injected with pICML1-rT16-1b and pBILE, but with low leak intensity. After induction, both the heart-specific rate and the intensity of transactivated GFP in pICMLM2_BILE-injected embryos were the same as those in embryos microinjected with the separated forms of plasmid (Table 2). Thus, we can conclude that the intensity of heart-specific GFP expression in the embryos microinjected with a single dual plasmid was not enhanced compared with the embryos microinjected with two plasmids, although the rate of heartspecific expression was slightly increased after Dox induction. 


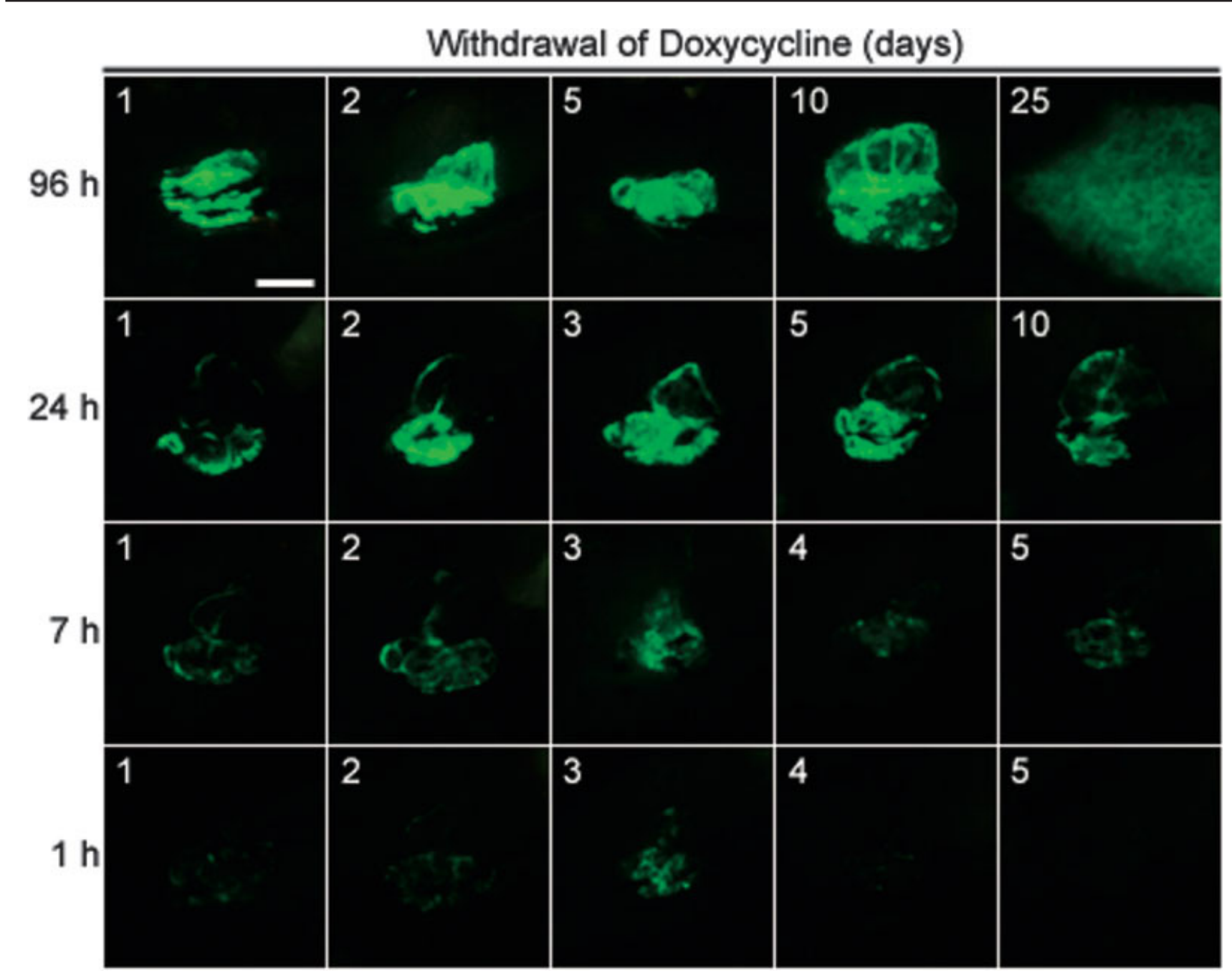

Fig. 4.

Addition of Dox (hours)

\begin{tabular}{l|l|l|l|l|}
\hline \hline- & 24 & 48 & 72 & 96 \\
& & & & \\
\hline
\end{tabular}

Withdrawal of Dox (days)

No Dox treatment

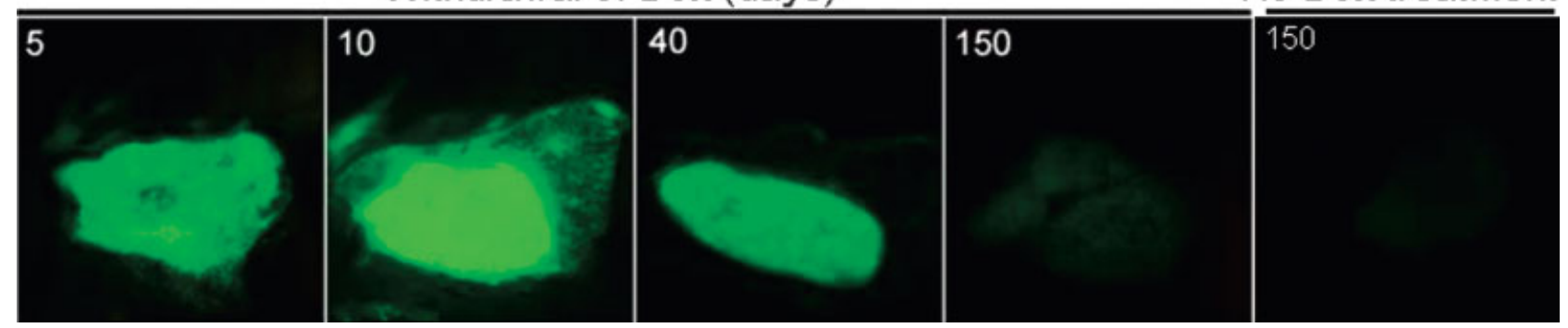

Fig. 5. 
Induced GFP Expression in the F2 Larvae Is Dependent on Dose and Duration of Dox Exposure

We generated two transgenic lines: T03 and T21. Line T03 was derived from embryos microinjected with plasmid pICML1-rt16-1b and plasmid pIBIE, whereas line T21 was derived from microinjection of the single dual pICMLM2_IBIE. The GFP expression was induced specifically in the heart of approximately $50 \%$ of siblings when the F2 3-days postfertilization (dpf) larvae derived from both line T03 and line T21 were treated with Dox in a concentration of $10 \mu \mathrm{g} / \mathrm{ml}$ for $12 \mathrm{hr}$ (Fig. 2A,B). Generally, the induced GFP expression was only slightly different among individuals of transgenic F2 siblings. There was no silencing of the transgene.

The dose-response and kinetics of Dox-mediated gene expression were measured by monitoring the time course of the GPF intensity. As shown in Figure 2C, Dox was ineffective for

Fig. 4. Green fluorescent protein (GFP) expression in heterozygotic F3 larvae derived from transgenic line T21 after withdrawing the inducer doxycycline (Dox). The intensity of GFP signal was expressed differently in 3-days postfertilization larvae exposed to $10 \mu \mathrm{g} / \mathrm{ml}$ of Dox for 1,7 , 24 , or $96 \mathrm{hr}$, as indicated. The number of days after Dox withdrawal is shown in the left corner on each panel. The GFP intensity increased continually for 2 or 3 days after Dox was withdrawn, then the level of the signal started to decrease. The greater the Dox exposure time, the higher the GFP expression level, and consequently, the more time needed to drop to the background level. Scale bar $=0.1 \mathrm{~mm}$.

Fig. 5. The photographic images of green fluorescent protein (GFP) appeared in the F3 adult fish after doxycycline (Dox) was added and then was withdrawn. After the 2-month-old adult fish derived from F3 of the transgenic line T21 were treated with Dox (+Dox) for the time indicated, the hearts were isolated and photographs of GFP expression were taken every $24 \mathrm{hr}$. After adult fish were treated with Dox for $96 \mathrm{hr}$, the fish were maintained in the absence of Dox and photographs of GFP expression were taken at the number of days indicated. Unexpectedly, the GFP expression increased for 10 days after Dox was withdrawn, and then the signal slowly decreased to the background level. The last panel shows that the 5-month-old adult fish (5-month) derived from the transgenic line T21 (T21) exhibited a relatively low background signal before induction. Scale bar $=0.5 \mathrm{~mm}$, except $0.1 \mathrm{~mm}$ for the last panel. induction at a concentration of less than $10 \mathrm{ng} / \mathrm{ml}$ and caused a weak GFP response at a concentration of $100 \mathrm{ng} / \mathrm{ml}$. However, the GFP intensity increased steadily when 1 and then $10 \mu \mathrm{g} / \mathrm{ml}$ Dox were used for induction. Of those two concentrations, $10 \mu \mathrm{g} / \mathrm{ml}$ was the most effective dose for induction. In addition, the intensity of induced GFP expression became stronger with the increased induction time of the $10 \mu \mathrm{g} / \mathrm{ml}$ Dox treatment, from 12 to $96 \mathrm{hr}$ starting in 3-dpf larvae (Fig. 2A-C).

\section{Transcriptions for GFP and rtTA-M2 Were Detected by Reverse Transcription- Polymerase Chain Reaction}

The mRNA transcripts of rtTA-M2 driven by a $c m l c 2$ promoter were detectable by using semiquantitative reverse transcription-polymerase chain reaction (RT-PCR) at the $12 \mathrm{hr}$ postfertilization (hpf) onward in F3 embryos derived from both line T03 and line T21 before induction (Fig. 3A). The RT-PCR signal reached a normal transcriptional level at $18 \mathrm{hpf}$ (Fig. 3A). Despite the presence of rtTA-M2 mRNA, the GFP transcripts were not detectable in the 3-dpf embryos (Fig. 3B). However, when these embryos were treated with Dox, a faint signal of GFP mRNA was detectable at $2 \mathrm{hr}$ after induction (Fig. 3B). This signal became clearly visible at $6 \mathrm{hr}$ after induction and reached a high expressional level at $12 \mathrm{hr}$ (Fig. 3B).

\section{Dox Exposure Time Required to Initiate GFP Expression}

F3 embryos were monitored using a fluorescence microscope at 2-hr intervals from $10 \mathrm{hpf}$ until induced GFP expression was observed. When 24-hpf F3 embryos were exposed to and kept in the presence of Dox, the earliest Dox-dependent GFP expression was observed at $31 \mathrm{hpf}$. Interestingly, when embryos younger than 24 hpf, such as $10,12,14,16,18,20$, and $22 \mathrm{hpf}$, were treated with Dox, GFP expression was not observable until at $31 \mathrm{hpf}$. However, the time required for GFP to appear decreased if embryos were dechorionated and then were treated with Dox. The GFP could be seen as early as 25-hpf when 18-hpf dechorionated embryos were treated with Dox. Meanwhile, when 72-hfp embryos were induced by Dox, the GFP was visible at $79 \mathrm{hpf}$. Therefore, we concluded that the duration of exposure required for detecting the reporter GFP fluorescence was $7 \mathrm{hr}$ after induction. Unexpectedly, when a wildtype female was immersed in Dox for 2 days before mating with an F2 homozygotic male, GFP expression was visible at 25 hpf in these F3 embryos.

\section{Dox Withdrawal Switches Off Induced GFP Expression in F3 Larvae and Adults}

Whereas a high level of GFP expression in the absence of Dox was apparent in some 5-month-old adults derived from transgenic line TO3, no such GFP background signal was found in any of the noninduced 13-dpf larvae or 5-monthold adult fish derived from the transgenic line T21. Therefore, we chose F3 larvae and adults derived from line T21 for performing the Dox withdrawal experiment.

To turn off the induced GFP expression in Dox-induced larvae, we stopped adding Dox to the culture water. If we treated the F3 3-dpf larvae derived of germ line T21 with Dox for only $1 \mathrm{hr}$, then within 5 days after Dox was removed from the water, the GFP signal was shut off (Fig. 4). However, the longer the Dox exposure, the more time (days) was needed for GFP expression to fade. For example, the heart still maintained induced GFP expression even 25 days after removal of Dox if the F3 3-dpf larvae had been treated with Dox for $96 \mathrm{hr}$, although the expression level of GFP was reduced slightly (Fig. 4). We also observed that the GFP signal was not reduced immediately when the Dox was removed. Instead, the intensity of the GFP fluorescence remained at a high level or even increased for 2 or 3 days after removal. We conclude that the time required to switch off the reporter gene in the induced larvae totally depended upon the length of exposure to Dox (Fig. 4).

After F3, 2-month-old adult fish derived from the transgenic line T21 were treated with Dox, the hearts were isolated, and photographs of GFP expression were taken every 24 hr. Results showed that the GFP re- 
porter gene was induced and expressed specifically in heart after exposure to Dox for $24 \mathrm{hr}$ (Fig. 5). The intensity of the GFP signal increased substantially after induction for $48 \mathrm{hr}$ (Fig. 5). On the other hand, when we withdrew Dox from the solution after the adult zebrafish had been treated with Dox for $96 \mathrm{hr}$, the GFP expression increased steadily for another 10 days after Dox withdrawal, and the GFP signal stayed at a relatively high level even 40 days after withdrawing Dox (Fig. 5). It took more than 150 days for GFP expression levels to return to background levels in the adults (Fig. 5). In conclusion, Dox treatment followed by Dox withdrawal demonstrated that GFP expression was tightly controlled by Dox also in adult fish: length of time of induction and the amount of time after Dox was withdrawal to reverse repression were much longer in adults.

\section{DISCUSSION}

In this study, we compared the luciferase activity of various transactivators, such as tTA, rtTA, rtTA-S2, rtTA-M2, rtTA2 ${ }^{\mathrm{S}}$-S2, and rtTA2 ${ }^{\mathrm{S}}-\mathrm{M} 2$, in zebrafish fibroblast cell lines (Table 1). Of the transactivators used in this study, tTA acts as a Tet-Off system, and the rest act as Tet-On systems. Unlike a previous study that demonstrated that the Tet-Off system is more effective than the Tet-On system in regulating transgene expression (Mizuguchi and Hayakawa, 2002), we found that rtTA-S2, rtTA-M2, and rtTA2 ${ }^{\mathrm{S}}$-S2 all have higher-fold induction than tTA. A further look at our results shows that rtTA-M2 and the humanized rtTA2 ${ }^{\mathrm{S}}$-S2 mutants have the best performance in terms of increase in luciferase activity after induction, but that rtTA-M2 can reach dramatically higher levels than rtTA2 ${ }^{\mathrm{S}}$-S2. These data suggest that prokaryotic codons of rtTA are favorable to human codons in zebrafish. This result is consistent with the Tet-On system as reported in Drosophila (Stebbins et al., 2001) and with the unicellular parasite $T$. gondii (Nakayama et al., 2000). However, this is not the case for HeLa cell line (Urlinger et al., 2000). The factors involved in this inconsistency remain to be determined in future studies. Be- cause rtTA-M2 displays the best inducible transactivator in the zebrafish cell line, we used this construct as an upstream plasmid to drive various rtTA-dependent downstream plasmids in a transgenic study.

To enhance the expression of an induced gene in the heart, we constructed plasmids in which the structural gene is flanked on both sides by AAV-ITR (Fu et al., 1998; Fig. 1). However, there is little difference after induction in terms of heart-specific rate and intensity of GFP expression in embryos microinjected either with or without the presence of AAV-ITR in the downstream reporter plasmids pIBIE and pBILE. This evidence suggests that AAV-ITR is not the mosteffective factor for enhancing the inducible expression in this system.

We attempted to reduce the leakiness as much as possible, while keeping the induced GFP intensity as high as possible. The transcription silencer tTS can be used to reduce the background level of the luciferase and for improving the regulation in the Tet-On system (Mizuguchi and Hayakawa, 2001). We constructed a downstream reporter plasmid pITSBIE, in which a transcriptional silencer is included (Fig. 1). Unfortunately, results showed that both the leak intensity and the induction intensity were reduced. Compared with embryos injected with pBILE, the induced GFP intensity was relatively faint in the embryos injected with pBILd2 (Table 2A). This finding may be explained by the fact that EGFP is more stable than d2EGFP, because the half-life of GFP is approximately $26 \mathrm{hr}$ (Corish and Tyler-Smith, 1999) and that of d2EGFP is approximately $2 \mathrm{hr}$ ( $\mathrm{Li}$ et al., 1998). As a reporter gene, enhanced GFP (EGFP) is easier to monitor than d2EGFP.

In the transient expression assay, we found that the heart-specific GFP expression rate of the embryos injected with the cointegrated form pICMLM2_IBIE was higher than that of the embryos co-microinjected with the two separate plasmids pICML1rT16-1b and pIBIE (Table 2). Based on the genetic inheritance analysis of the F2 offspring derived from line T03, we conclude that there is only a single insertion site for pICML1-rT16-1b and pIBIE in the host chromosome.
Concatamerization of transferred DNA fragments has been reported in transgenic fish (Yang et al., 1999; Hsiao et al., 2001). We speculate that concatamerization may occur between these two plasmids before integration into the chromosome of the host cells. Unlike the cointegrated form of plasmid pICMLM2_IBIE, the ratio of rtTA-M2 gene to IBIE gene in the concatamer of pICML1-rT16-1b and pI$\mathrm{BIE}$ is unclear.

Because it is rather difficult with the naked eye to discern intensity when the signal strength of the GFP is high, we used a digital camera with KODAK 1D Image Analysis Software (software for analyzing the results of gel electrophoresis) to quantify the GFP signal shown on the photographic images. For accurate quantification of fluorescent signals in living cells, we considered both the fluorescence and the structural properties of the GFP, following the basic photophysical characteristics for determining brightness (Patterson et al., 1997). As all the photographs were taken under the same conditions, the structural properties exerted by the GFP, being uniform for each sample, thus could be disregarded. The images, therefore, can be compared directly to assess the GFP content. The higher the image intensity we observed, the higher the GFP content we obtained. Using this software, we took the average intensity of the pixels of the defined area (mean intensity) as an index to quantify the amount of fluorescent signals occurring in the heart after induction, as shown in Figure 2. The quantification is useful for making a comparison of the GFP expression in similar developmental stages.

As shown in the RT-PCR experiment (Fig. 3A), the transcript of rtTA-M2 is detectable at $12 \mathrm{hpf}$, which is $4 \mathrm{hr}$ earlier than previously reported by whole-mount in situ hybridization (Huang et al., 2003). The time course of Dox induction was investigated by examining the images of the GFP. Of interest, we found that, no matter what stage before $18 \mathrm{hpf}$ of the dechorionized embryos being induced, the transactivated GFP cannot be imaged until $25 \mathrm{hpf}$. On the other hand, when Dox was added to any stage after $18 \mathrm{hpf}$, GFP became visible after 
induction for $7 \mathrm{hr}$. This result is consistent with embryos originating from parental females that are treated with Dox for 3 days. On the basis of the semiquantitative RT-PCR results shown in Figure 3A, the rtTA-M2 mRNA level increases dramatically at $18 \mathrm{hpf}$. Therefore, it is reasonable to conclude that the molecules of rtTA-M2 are not transcribed sufficiently to transactivate the downstream GFP gene until $18 \mathrm{hpf}$. We also note that the GFP is not visible until $31 \mathrm{hpf}$ if the induction is carried out on embryo whose chorion has not been removed. We speculate that the chorion may be a barrier for transferring Dox, resulting in a delay of induction. In fact, the GFP mRNA is detectable by RT-PCR after being induced for 2 hr (Fig. 3B), although the GFP cannot yet be observed until $7 \mathrm{hr}$ after induction. We conclude that the time required to transactivate the GFP reporter in this system is $7 \mathrm{hr}$ after induction but that the strength of the promoter and the existence of the chorion affect the efficiency of the zebrafish Tet-On system.

In a study of the tTA system in the heart of transgenic mice, the reporter luciferase gene was nonspecifically apparent in the kidney, lung, and tongue (Yu et al., 1996). However, in the system we developed, the GFP reporter gene is transactivated specifically in the heart of the heterozygous F3 individuals derived of line T21 without showing any leaky expression of GFP. Urlinger et al. (2000) reported that rtTA has a distinct residual affinity toward tet $\mathrm{O}$ and produces background activity of $\mathrm{P}_{\text {tet }}-1$ when rtTA is present in relatively high intracellular concentrations. The transgenic lines in this study, T03 and T21, present different levels of background GFP expression, indicating that the microinjection of a cointegrated form of plasmid is superior to the microinjection of dual plasmids in terms of minimal leakiness of the GFP.

When the Tet-On system is carried out in cell lines, the inducer is immersed in the medium and enters directly into the cells. The activation of a transgene can reach a maximum level after induction for $24 \mathrm{hr}$ (Gossen et al., 1995). However, when the Tet-On system is carried out on transgenic fish, the fluorescent signal in the derived larvae cannot reach the maximal level, even after induction for 96 hr (Fig. 2C). In adult fish, this time course is extended further, not only in the length of time of Dox induction but also in that of Dox withdrawal. The longer time that is needed indicates that the inducer needs to be transported to the heart of the fish through the circulatory system, rather than through simple diffusion.

It takes more than 25 days (Fig. 4) or 100 days (Fig. 5) to abolish the GFP expressed in induced larvae and adult fish, respectively, after Dox withdrawal. One of the possible reasons is that the concentration of Dox located at the inter- and intracellular level remains at a rather high level, even after the Dox is withdrawn. The GFP expression in embryos generated by crossing the Dox-incubated wild-type female with the transgenic T03 male provides evidence to support the idea that the GFP signal appears in these embryos at $25 \mathrm{hpf}$ without any Dox treatment. The image is similar to that of the Dox-treated and dechorionated embryos. Nevertheless, this feature could be beneficial in some cases, such as in an animal model of disease in which a transgenic product that lasts longer after induction is desirable.

Currently, zebrafish has become an important vertebrate model organism, because this species can be used to study human diseases (Hsieh and Liao, 2002; Shin and Fishman, 2002), physiology (Farber et al., 2001), and pharmacology (Langherinrich, 2003). We successfully developed a Tet-On system in zebrafish that enables the transgene to be expressed specifically in the heart after induction. This system may be applied in the investigation of the mechanism of the function and development of the heart, in the model of cardiovascular disease, and in the screening of drugs for the treatment of heart disease.

\section{EXPERIMENTAL PROCEDURES}

\section{Cell Line and Luciferase Assay}

The zebrafish fibroblast cell-line ZEM2S (ATCC, CRL-2147) was cultured with L-15 complete medium: 50
\% L-15 medium (ATCC 30-2008) supplemented with $35 \%$ DMEM (high glucose, GIBCO 12100), $15 \%$ F12 medium (GIBCO 21700), $0.18 \mathrm{~g} / \mathrm{L}$ sodium bicarbonate, $15 \mathrm{mM}$ HEPES, and $10 \%$ heat-inactivated fetal bovine serum. The explants were incubated at $28^{\circ} \mathrm{C}$, without extracting $\mathrm{CO}_{2}$. The cells were cotransfected with the plasmid containing a transactivator, such as tTA, rtTA, rtTA-S2, rtTA-M2, rtTA2 ${ }^{\mathrm{S}}$ $\mathrm{S} 2$, or rtTA2 ${ }^{\mathrm{S}}-\mathrm{M} 2$ (Urlinger et al., 2000), controlled by the CMV promoter and the plasmid pUHC13-3 (Gossen and Bujard, 1992), containing a luciferase reporter gene driven by the Tet-responsive element (TRE). Cells were grown in the absence or the presence of $1 \mu \mathrm{g} / \mathrm{ml}$ Dox for $40 \mathrm{hr}$ and then harvested for dual luciferase assays (Promega), which were carried out according to the recommendations of the manufacturer. Luciferase activity was measured from the cell extracts and normalized to Renila luciferase activities expressed from pRL-TK included in the transfection mixture.

\section{Plasmid Constructs}

The six constructs used in this study are illustrated schematically in Figure 1 . The cardiac-specific rtTA construct pICML1-rT16-1b was generated by modifying the previously described pICMLE-(-870/787) plasmid (Huang et al., 2003), in which the upstream region (-870/-1), exon 1 , intron 1 , and a part of exon 2 of zebrafish cmlc2 were included. The EGFP segment of pICMLE-(-870/ 787) was replaced by rtTA-M2. The resulting pICML1-rT16-1b consisted of the regulatory region of $c m l c 2$, rtTA-M2, and AAV-ITR. The rtTA-dependent bidirectional tet $\mathrm{O}$ (Clontech) was cloned into pIEGFP (Chou et al., 2001) to generate pIBIE, resulting in TRE being located upstream of the EGFP gene. Plasmid pITSBIE followed a similar construction to that of pIBIE, except that a Tet-controlled transcriptional silencer (Clontech) was included. Plasmids pBILE and pBILd2 were generated by cloning EGFP and d2EGFP cDNA into pBIL (Clontech), respectively. Luciferase and EGFP were engineered downstream on each side of bidirectional tet $\mathrm{O}$ in the absence of AAV-ITR. 
Another strategy of plasmid construction used in this study was the cointegrated form of a plasmid containing the cardiac-specific rtTA and a plasmid containing the rtTA-dependent GFP reporter gene. Plasmid pICML1-rT16-1b was fused with plasmid pIBIE or pBILE to generate a single dual plasmid of pICMLM2_IBIE or pICMLM2_BILE, respectively.

\section{Experimental Fish and GFP Observations}

Zebrafish of the AB strain were cultured and maintained according to the procedures described by Westerfield (1995). The developmental stages were determined according to criteria described by Kimmel et al. (1995). One-cell stage fertilized eggs were collected and microinjected with linearized constructs (Wang et al., 2002). A total of $15 \mu \mathrm{g} / \mathrm{ml}$ of each plasmid was microinjected. Injected embryos were incubated at $28.5^{\circ} \mathrm{C}$. Transgenic embryos were observed under a stereodissecting microscope (Leica MZFIII) equipped with a fluorescent module and an enhanced EGFP filter cube (Kramer Scientific). We categorized the level of GFP intensity by using stars: the highest expression was designated $* * * * *$, whereas the lowest was designated $*$.

\section{Transgenic Germ Lines, Induction, and GFP Detection}

The embryos microinjected with pICML1-rt16-1b and pIBIE were cultured under a 14-hr light and 10-hr dark photoperiod at $28.5^{\circ} \mathrm{C}$. Pairs of mature fish were kept together in tanks, and their eggs were collected for study. Transgenic embryos were monitored for GFP expression after induction with $10 \mu \mathrm{g} / \mathrm{ml}$ of Dox (Sigma) for 3 days, after which the GFP-positive progeny were bred.

The F2 embryos derived from the transgenic germ lines were kept in water until $72 \mathrm{hpf}$, then they were treated with $10 \mu \mathrm{g} / \mathrm{ml}, 1 \mu \mathrm{g} / \mathrm{ml}, 100$ $\mathrm{ng} / \mathrm{ml}$, or $10 \mathrm{ng} / \mathrm{ml}$ of Dox. Individual larvae, six larvae from each concentration, were kept in individual wells of 24-well culture plate. After induction, GFP expression was observed under the fluorescence microscope.
Fish were orientated ventrally and tilted at 45 degrees when taking photos using a Coolpix 990 digital camera (Nikon) with 10-sec exposure time at ISO 400. The photographic image of the GFP intensity was analyzed by using KODAK 1D Image Analysis Software. An area for analysis was defined by using the Free Form Tool in the ROI Toolbar. The mean intensity of a selected area from the ROI information window was recorded by quantifying the strength of the GFP fluorescent signal. Fluorescence intensity was processed and quantified using by Microsoft Excel. For every larva, one set of photos was taken at 12-hr intervals, from before Dox exposure until 96-hr after Dox treatment. If the fish died before the $96-\mathrm{hr}$ period was up, the photos were not used in the analysis.

\section{Strategies for Dox Induction}

Three methods were used to induce GFP expression after the $10 \mu \mathrm{g} / \mathrm{ml}$ Dox treatment. (1) Immersion of a female: a wild-type female was immersed in Dox for 2 days and crossed with an F2 homozygotic male. (2) Immersion of embryos: F3 embryos were incubated with Dox at different developmental stages at 2-hr intervals from $10 \mathrm{hpf}$ to $24 \mathrm{hpf}$. (3) Immersion of dechorionated embryos: same procedure as for method 2 except that the chorion of the embryo was removed. The GFP expression in the embryos was monitored by using a fluorescence microscope at 2-hr intervals until GFP was observed.

\section{RT-PCR}

The RNA of the embryos at different stages was isolated using Trizol (ProTech) and treated with RNase-free DNase (Promega). RT was carried out using Moloney-murine leukemia virus (M-MLV; Promega) reverse transcriptase with $2 \mu \mathrm{g}$ of total RNA in a volume of $25 \mu \mathrm{l}$. PCR was then performed using $1 \mathrm{U}$ of VioTag (Viogene) and $1 \mu \mathrm{l}$ of the $\mathrm{RT}$ product per $50-\mu \mathrm{l}$ reaction for 35 cycles at an annealing temperature of $62^{\circ} \mathrm{C}$ for EGFP detection, and at $54^{\circ} \mathrm{C}$ for rtTA M2 detection. Primers used for detecting EGFP gene were EGFP-404F, ACGACGGCAACTACAAGACCCGC, and EGFP-814R,
ACTTGTACAGCTCGTCCATGCCGA, whereas primers for detecting rtTA-M2 gene were rT16-1F4, ATTACGGGTCTACCATCGAGGGC, and rT16-1R5, CCCTACCCACCGTACTCGTACAAT. Primers used for detecting the $\beta$-actin gene, which acted as an internal control, were BA-F, CGGATTCGCTGGAGATGATGC, and BA-R, CGGAGGATGGCATGGGGCA. The PCR reaction was carried out at an annealing temperature of $66^{\circ} \mathrm{C}$ for 25 cycles. The sizes of the expected PCR products were 410, 340, and 473 bp for genes of EGFP, rtTA-M2, and $\beta$-actin, respectively.

\section{ACKNOWLEDGMENTS}

We thank W. Hillen for the gift of pUHC13-3 and the construct encoding rtTA2 ${ }^{\mathrm{S}}$-M2. H.J.T. and T.S.J. were funded by the National Science Council of Taiwan, and F.J.H. was funded by a grant from the National Taiwan University Hospital.

\section{REFERENCES}

Chou CY, Hong LS, Tsai HJ. 2001. Uniform GFP-expression in transgenic medaka (Oryzias latipes) at the F0 generation. Transgenic Res 10:303-315.

Corish P, Tyler-Smith C. 1999. Attenuation of green fluorescent protein half-life in mammalian cells. Protein Eng 12:10351040.

Farber SA, Pack M, Ho SY, Johnson ID, Wagner DS, Dosch R, Mullins MC, Hendrickson HS, Hendrickson EK, Halpern ME. 2001. Genetic analysis of digestive physiology using fluorescent phospholipid reporters. Science 292:1385-1388.

Fishman MC, Chien KR. 1997. Fashioning the vertebrate heart: earliest embryonic decisions. Development 124:2099-2117.

Fishman MC, Stainier DY. 1994. Cardiovascular development. Prospects for a genetic approach. Circ Res 74:757-763.

Fu Y, Wang Y, Evans SM. 1998. Viral sequences enable efficient and tissue-specific expression of transgenesis in Xenopus. Nat Biotechnol 16:253-257.

Furth PA, St Onge L, Boger H, Gruss P, Gossen M, Kistner A, Bujard H, Hennighausen L. 1994. Temporal control of gene expression in transgenic mice by a tetracycline-responsive promoter. Proc Natl Acad Sci U S A 91:9302-9306.

Gossen M, Bujard H. 1992. Tight control of gene expression in mammalian cells by tetracycline-responsive promoters. Proc Natl Acad Sci U S A 89:5547-5551.

Gossen M, Freundlieb S, Bender G, Muller G, Hillen W, Bujard H. 1995. Transcriptional activation by tetracycline in mammalian cells. Science 268:1766-1769. 
Hsiao CD, Hsieh FJ, Tsai HJ. 2001. Enhanced expression and stable transmission of transgenes flanked by inverted terminal repeats from adeno-associated virus in zebrafish. Dev Dyn 220:323-336.

Hsieh DJ, Liao CF. 2002. Zebrafish M2 muscarinic acetylcholine receptor: cloning, pharmacological characterization, expression patterns and roles in embryonic bradycardia. Br J Pharmacol 137: 782-792.

Huang CJ, Tu CT, Hsiao CD, Hsieh FJ, Tsai HJ. 2003. Germ-line transmission of a myocardium-specific GFP transgene reveals critical regulatory elements in the cardiac myosin light chain 2 promoter of zebrafish. Dev Dyn 228:30-40.

Jost M, Kari C, Rodeck U. 1997. An episomal vector for stable tetracycline-regulated gene expression. Nucleic Acids Res 25:3131-3134.

Kimmel CB, Ballard WW, Kimmel SR, Ullmann B, Schilling TF. 1995. Stages of embryonic development of the zebrafish. Dev Dyn 203:253-310.

Kistner A, Gossen M, Zimmermann F, Jerecic J, Ullmer C, Lubbert H, Bujard H. 1996. Doxycycline-mediated quantitative and tissue-specific control of gene expression in transgenic mice. Proc Natl Acad Sci U S A 93:10933-10938.

Langheinrich U. 2003. Zebrafish: a new model on the pharmaceutical catwalk. Bioessays 25:904-912.

Li X, Zhao X, Fang Y, Jiang X, Duong T, Fan C, Huang CC, Kain SR. 1998. Generation of destabilized green fluorescent protein as a transcription reporter. J Biol Chem 273:34970-34975.

Meissner M, Brecht S, Bujard H, Soldati D. 2001. Modulation of myosin A expression by a newly established tetracycline repressor-based inducible system in Toxoplasma gondii. Nucleic Acids Res 29:e115.

Mizuguchi H, Hayakawa T. 2001. Characteristics of adenovirus-mediated tetracy- cline- controllable expression system. Biochim Biophys Acta 1568:21-29.

Mizuguchi H, Hayakawa T. 2002. The tetoff system is more effective than the tet-on system for regulating transgene expression in a single adenovirus vector. $\mathrm{J}$ Gene Med 4:240-247.

Nakayama H, Mio T, Nagahashi S, Kokado M, Arisawa M, Aoki Y. 2000. Tetracycline-regulatable system to tightly control gene expression in the pathogenic fungus Candida aibicans. Infect Immun 68:6712-6719.

Passman RS, Fishman GI. 1994. Regulated expression of foreign genes in vivo after germline transfer. J Clin Invest 94:24212425.

Patterson GH, Knobel SM, Sharif WD, Kain SR, Piston DW. 1997. Use of the green fluorescent protein and its mutants in quantitative fluorescence microscopy. Biophys J 73:2782-2790.

Poss KD, Wilson LG, Keating MT. 2002. Heart regeneration in zebrafish. Science 298:2188-2190.

Raya AKC, Koth CM, Buscher D, Kawakami Y, Itoh T, Raya RM, Sternik G, Tsai HJ, Rodriguez-Esteban C, and Izpisua-Belmonte JC. 2003. Activation of notch signaling pathway precedes heart regeneration in zebrafish. Proc Natl Acad Sci U S A 100:11889-11895.

Salucci V, Scarito A, Aurisicchio L, Lamartina S, Nicolaus G, Giampaoli S, Gonzalez-Paz O, Toniatti C, Bujard H, Hillen W, Ciliberto G, Palombo F. 2002. Tight control of gene expression by a helperdependent adenovirus vector carrying the $\operatorname{rtTA2}(\mathrm{s})-\mathrm{M} 2$ tetracycline transactivator and repressor system. Gene Ther 9:1415-1421.

Sehnert AJ, Huq A, Weinstein BM, Walker C, Fishman M, Stainier DY. 2002. Cardiac troponin $\mathrm{T}$ is essential in sarcomere assembly and cardiac contractility. Nat Genet 31:106-110.

Shin JT, Fishman MC. 2002. From Zebrafish to human: modular medical mod- els. Annu Rev Genomics Hum Genet 3: 311-340.

Shu X, Cheng K, Patel N, Chen F, Joseph E, Tsai HJ, Chen JN. 2003. Na,K-ATPase is essential for embryonic heart development in the zebrafish. Development 130:6165-6173.

Stebbins MJ, Urlinger S, Byrne G, Bello B, Hillen W, Yin JC. 2001. Tetracycline-inducible systems for Drosophila. Proc Natl Acad Sci U S A 98:10775-10780.

Urlinger S, Baron U, Thellmann M, Hasan MT, Bujard H, Hillen W. 2000. Exploring the sequence space for tetracycline-dependent transcriptional activators: novel mutations yield expanded range and sensitivity. Proc Natl Acad Sci U S A 97:7963-7968.

Wang TM, Chen YH, Liu CF, Tsai HJ. 2002. Functional analysis of the proximal promoter regions of fish rhodopsin and $m y f-5$ genes using transgenesis. Mar Biotechnol 4:247-255.

Weinmann P, Gossen M, Hillen W, Bujard H, Gatz C. 1994. A chimeric transactivator allows tetracycline-responsive gene expression in whole plants. Plant J 5: 559-569.

Westerfield M. 1995. The Zebrafish Book, Eugene: University of Oregon press. p $1.1-3.25$.

Yang J, Zhou W, Zhang Y, Zidon T, Ritchie T, Engelhardt JF. 1999. Concatamerization of adeno-associated virus circular genomes occurs through intermolecular recombination. J Virol 73:9468-9477.

Yu Z, Redfern CS, Fishman GI. 1996. Conditional transgene expression in the heart. Circ Res 79:691-697.

Zhu Z, Zheng T, Lee CG, Homer RJ, Elias JA. 2002. Tetracycline-controlled transcriptional regulation systems: advances and application in transgenic animal modeling. Semin Cell Dev Biol 13:121128. 